\title{
Review
}

\section{Human T-cell lymphotropic virus type 1 and its oncogenesis}

\author{
Lan-lan ZHANG ${ }^{1}$, Jing-yun WEI ${ }^{1}$, Long WANG ${ }^{1}$, Shi-le HUANG ${ }^{2}$, Ji-long $\mathrm{CHEN}^{1,3,4, *}$ \\ ${ }^{1}$ Key Laboratory of Fujian-Taiwan Animal Pathogen Biology, College of Animal Sciences, Fujian Agriculture and Forestry University, \\ Fuzhou 350002, China; ${ }^{2}$ Department of Biochemistry and Molecular Biology, Louisiana State University Health Sciences Center, \\ Shreveport, LA, USA; ${ }^{3}$ CAS Key Laboratory of Pathogenic Microbiology and Immunology, Institute of Microbiology, Chinese Academy of \\ Sciences, Beijing 100101, China; ${ }^{4}$ University of Chinese Academy of Sciences, Beijing 100049, China
}

\begin{abstract}
Human T-cell lymphotropic virus type 1 (HTLV-1) is the etiologic agent of adult T-cell leukemia/lymphoma (ATL), a rapidly progressing clonal malignancy of $\mathrm{CD} 4^{+} \mathrm{T}$ lymphocytes. Exploring the host-HTLV-1 interactions and the molecular mechanisms underlying HTLV-1mediated tumorigenesis is critical for developing efficient therapies against the viral infection and associated leukemia/lymphoma. It has been demonstrated to date that several HTLV-1 proteins play key roles in the cellular transformation and immortalization of infected T lymphocytes. Of note, the HTLV-1 oncoprotein Tax inhibits the innate IFN response through interaction with MAVS, STING and RIP1, causing the suppression of TBK1-mediated phosphorylation of IRF3/IRF7. The HTLV-1 protein HBZ disrupts genomic integrity and inhibits apoptosis and autophagy of the target cells. Furthermore, it is revealed that HBZ enhances the proliferation of ATL cells and facilitates evasion of the infected cells from immunosurveillance. These studies provide insights into the molecular mechanisms by which HTLV-1 mediates the formation of cancer as well as useful strategies for the development of new therapeutic interventions against ATL. In this article, we review the recent advances in the understanding of the pathogenesis, the underlying mechanisms, clinical diagnosis and treatment of the disease caused by HTLV-1 infection. In addition, we discuss the future direction for targeting HTLV-1-associated cancers and strategies against HTLV-1.
\end{abstract}

Keywords: human T-cell lymphotropic virus type 1; adult T-cell leukemia/lymphoma; Tax; HBZ; tumorigenesis; innate immunity

Acta Pharmacologica Sinica (2017) 38: 1093-1103; doi: 10.1038/aps.2017.17; published online Apr 102017

\section{Introduction}

Human T-cell leukemia viruses (HTLVs), belonging to the primate T-lymphotropic virus (PTLV) family ${ }^{[1]}$, are complex retroviruses. HTLVs are classified as the Delta-retroviruses genera of the Orthoretrovirinae subfamily ${ }^{[2]}$. Approximately 30000 40000 years ago, simian T-lymphotropic viruses (STLVs) were transmitted from monkeys to humans in Africa and gradually evolved into HTLVs. Since then, HTLVs have spread to different geographic regions with human migration ${ }^{[3]}$. Nevertheless, STLVs, having high homology to HTLVs, still exist in Africa ${ }^{[4]}$. To date, four types of HTLVs, ie, HTLV1/2/3/4, have been identified. HTLV-1, the first oncogenic HTLV in humans, was originally discovered in the early 1980s by two independent research groups in America and Japan ${ }^{[2,5]}$. The virus predominantly infects $\mathrm{T}$ lymphocytes, and its genome is composed of a positive single-stranded RNA ${ }^{[6]}$. HTLV-1, including its seven

\footnotetext{
* To whom correspondence should be addressed.

E-mail chenjl@im.ac.cn

Received 2016-12-13 Accepted 2017-02-27
}

subtypes (HTLV-1A to $-1 G$ ), is endemic in Japan, Melanesia, Iran, Central and West Africa, the Caribbean, Australia, and the Middle East ${ }^{[7,8]}$. It is estimated that approximately 10 million people are infected with HTLV-1 around the world, but most of them remain asymptomatic throughout the rest of their lives ${ }^{[7]}$. Only a small proportion (2\%-5\%) of HTLV1-infected humans progress to adult T-cell leukemia/lymphoma (ATL), a very aggressive form of leukemia ${ }^{[9]}$. Another important HTLV-1-associated disease is HAM/TSP (HTLVassociated myelopathy/tropical spastic paraparesis), a type of neurological demyelinating disease ${ }^{[10,11]}$. In addition, three other genotypes of HTLVs, namely, HTLV-2, HTLV-3, and HTLV-4, have been isolated and characterized ${ }^{[12]}$. HTLV-1 and HTLV-2 are more prevalent than HTLV-3 and HTLV-4 ${ }^{[12]}$. Although the CD4 ${ }^{+}$T-cell are the main target cells of HTLV-1, many other types of cells, such as $\mathrm{CD}^{+} \mathrm{T}$-cell, endothelial cells, B-lymphocytes, myeloid cells, and fibroblasts, can also be infected with HTLV-1 ${ }^{[13,14]}$. Therefore, HTLV-1 is a significant threat to public health. In this article, we highlight the 
current knowledge of HTLV-1, including HTLV-1-mediated oncogenesis, the critical proteins encoded by HTLV-1 and the mechanisms underlying their functions during viral infection. In addition, we also review the strategies for the prevention of HTLV-1-associated cancer and the future direction of cancer research in the field of HTLV-1.

\section{Genotypes of HTLVs}

Four genotypes of HTLVs have been found as described above. HTLV-1 can be transmitted via body fluids, e.g., blood, breast milk and semen ${ }^{[15]}$. Transmission from mother to child occurs primarily via breast-feeding and rarely through transplacental or intrapartum transmission in endemic regions ${ }^{[16,17]}$. In most cases, sexual transmission occurs from a man to a woman during sexual intercourse ${ }^{[15-17]}$. Moreover, human-to-human parallel transmission, primate-to-human transmission, and nonhuman transmission have also been observed ${ }^{[18]}$.

Previous studies have sought to determine the mechanism underlying the oncogenesis of HTLV-1. It has been shown that HTLV-1, unlike other acute transforming retroviruses, is neither able to induce tumors rapidly nor able to upregulate the expression of cellular proto-oncogenes ${ }^{[19]}$. In general, viral infections can initiate and promote tumorigenesis by any of the following mechanisms: induction of chronic inflammation in the host, suppression of the host immune defense, insertional mutation in the host genome and activation of virally carried oncogenes that mediate cellular transformation ${ }^{[20-22]}$. Studies have demonstrated that HTLV-1-induced oncogenesis may involve multiple mechanisms ${ }^{[19,23,24]}$. First, a prolonged and persistent infection with HTLV-1 results in chronic inflammation in the host. Then, phagocytes release reactive oxygen species at the inflammatory site, which interact with nitrogen radicals, causing damage to the cell membrane, DNA and proteins and altering the gene expression profiles and enzymatic activities. Consequently, these responses induce carcinogenesis and enhance neoplasia ${ }^{[23]}$. Second, HTLV-1 infection can cause the insertion of efficient oncogenes into the host genome, which reduce the expression of tumor suppressor genes or directly stimulate mitosis of the host cells ${ }^{[24]}$. Third, HTLV-1 infection can also inhibit the host immune function and impair immune surveillance, and thus, pre-cancerous cells can escape from host immunity ${ }^{[24]}$. However, novel insights into the precise mechanisms by which HTLV-1 induces tumorigenesis are expected to be discovered in the future using new approaches.

Importantly, it is thought that HTLV-1 infection happens early in life and has a long latency period before cancer appears. Thus, the vast majority of effort has focused on the prevention of HTLV-1-mediated tumorigenesis. Because the exposure to HTLV-1 in early life plays an important role in cancer development later in life, it is practical to develop interventions targeting early life exposure to the infection ${ }^{[25]}$.

HTLV-2 was initially described in $1982^{[26]}$. Molecular biology experiments have defined four major subtypes of HTLV-2, namely, HTLV-2A/B/C/D ${ }^{[27]}$. HTLV-2 is endemic within the Amerindian and Pygmy populations and epidemic among intravenous drug users, with 3-5 million cases worldwide ${ }^{[28]}$.
HTLV-2 is relatively less pathogenic than HTLV-1. Occasionally, HTLV-2 infection ends in sub-acute neurological syndromes, such as neuropathies and paraparesis, despite being very rare ${ }^{[29,30]}$.

Human HTLV-3 and HTLV-4 are two new members of the HTLV family. In 2005, Gessain's group and others reported the discovery of the human HTLV-3 in two asymptomatic inhabitants of South Cameroon. A fourth HTLV type (HTLV-4) was also discovered in the same geographical area ${ }^{[31,32]}$. Interestingly, recent molecular biology investigations have shown that HTLV-4 lineage is older than the ancestors of HTLV-1, HTLV-2 and HTLV- $3^{[33]}$. Although a number of distinguishing characteristics of HTLV-3 and HTLV-4 have been revealed, the pathogenic potential of these human retroviruses remains to be determined.

Notably, the original name for human immunodeficiency virus (HIV), the virus isolated by Gallo's team in 1984, was HTLV-III ${ }^{[34]}$, but it has since fallen out of use. Collectively, it was demonstrated that HIV was the cause of a new epidemic, AIDS $^{[35]}$. Like HTLV-1, similar risk factors were observed for HIV-1 infection, including blood exposure, sexual contact, and birth to a mother with the disease ${ }^{[36]}$. Both HTLV-1 and HIV-1 target cells of the immune system, particularly CD4+ T-cell, and cause them to grow abnormally (HTLV-1) or die (HIV-1). In addition, they encode regulatory proteins essential for viral replication, such as TAX, Rex (HTLV-1), and Tat, Rev (HIV-1) ${ }^{[35,37]}$. However, although HTLV-1 and HIV-1 share similar routes of transmission, they cause significantly different diseases. ATL is the major disease caused by HTLV-1 infection, while infection with HIV-1 ultimately results in deficiency of the human immune system.

\section{Biological structure of HTLVs and their components}

Similar to other retroviruses, HTLV-1 begins its life cycle by targeting specific cells. HTLV-1 contains an RNA genome in the virion ${ }^{[38]}$. Upon viral entry, the viral RNA is reverse transcribed into a double-stranded DNA, which can be integrated into the DNA genome of the host cell, resulting in the provirus ${ }^{[39]}$. HTLV-1 has a relatively small $(9 \mathrm{~kb})$ genome, but it can express multiple products by utilizing various strategies, including polycistronic translation, frame shifting, alternative mRNA splicing, and protease-mediated cleavage of large viral proteins into smaller polypeptides with specific functions ${ }^{[40]}$.

All four HTLVs have similar genomic structures, as demonstrated by comparative studies of their genomic sequences ${ }^{[1]}$. HTLV-1 and HTLV-2 have common molecular features and exhibit approximately $70 \%$ nucleotide sequence homology ${ }^{[28]}$. HTLV-3 has approximately $62 \%$ nucleotide sequence homology with HTLV-1, and HTLV-4 shares approximately $62 \%-$ $71 \%$ sequence identity with HTLV-1, HTLV-2, and HTLV- $3{ }^{[41]}$. However, there is one major difference between the nucleotide sequences of HTLV-1/2 and HTLV-3/4: the long terminal repeats (LTRs) of HTLV-3/4 lack the distal 21-bp transcription regulatory repeat sequences, $i e$, Tax-responsive elements (TREs), and possess only two 21-bp repeat sequences, while the LTRs of HTLV-1/2 have three of these 21-bp repeat 
sequences ${ }^{[41,42]}$. In addition to the two LTR-containing cisacting regulatory sequences of the provirus, all four HTLV genotypes contain the structural genes gag, pro, pol, and env and the regulatory genes tax and $r e x^{[38]}$ (Figure 1).

One region (named PX) located downstream of the envelope gene of HTLVs contains several open reading frames (ORFs) encoding auxiliary proteins. HTLV-1 encodes four different auxiliary proteins, including p13, p30, p12 and p8 ${ }^{[43]}$ (Figure 1). These proteins play crucial roles during HTLV-1 infection. p30 decreases the expression of Tax, a viral transcription transactivator, thus promoting viral latency and persistence and helping the virus escape from immune surveillance ${ }^{[44]}$. Similar to p30, p13 also enables the virus to bypass immune surveillance by increasing the production of mitochondrial ROS (reactive oxygen species) ${ }^{[45]}$. As a result, p13 may promote viral latency and persistence ${ }^{[46]}$. p12 inhibits the activation of cytotoxic T lymphocytes (CTLs) and natural killer cells $(\mathrm{NK})$, hence protecting the virus against immune surveillance. p8, a proteolytic cleavage product of p12, accelerates T-cell contact by augmenting the number and length of cellular conduits among $\mathrm{T}$-cell and enhances the envelope-dependent transmission of $\mathrm{HTLV}-\mathrm{1}^{[47]}$. In addition to the proteins $\mathrm{p} 13$ and p30, HTLV-2 also encodes regulatory and accessory proteins, such as p10, p11 and p28. Given the genomic features of HTLVs, more potential ORFs encoding auxiliary proteins may be discovered in the future ${ }^{[1]}$. For example, HTLV-2 contains a potential ORF called APH-2 on its antisense strand, which is analogous to HTLV-1 bZIP factor (HBZ), an antisense transcript of the HTLV-1 provirus ${ }^{[48,49]}$, as well as APH-3/4 of HTLV-3/ $4^{[50]}$. Sequence analysis has demonstrated that APH-3 and APH-4 are more closely related to APH-2 than to HBZ ${ }^{[50]}$. APH-2, APH-3 and APH-4 do not contain a bZIP (basic leucine zipper) domain, which is present in HBZ. Additionally, APH-2, APH-3 and APH-4 differ from HBZ in their subcellular localization ${ }^{[48,50]}$. The Tax gene, located between env and 3'-LTR, is encoded within the pX region of HTLVs and is highly conserved among four genotypes and different subtypes of HTLVs ${ }^{[51]}$. The structural and functional domains of Tax-1, Tax-2, Tax-3, and Tax-4 are shown in Figure 1.

\section{Invasion of HTLV-1 and HTLV-1-mediated cellular trans- formation}

The invasion of HTLV-1 marks the beginning of the viral survival and replication ${ }^{[52]}$. HTLV-1 binds to its receptor on the target cells, which initiates the invasion process ${ }^{[52]}$. The receptor complex is composed of glucose transporter (GLUT1), heparin sulfate proteoglycan (HSPG) and the VEGF-165 receptor neuropilin-1 (NRP-1) ${ }^{[53,54]}$. HTLV-1 interacts with HSPG,
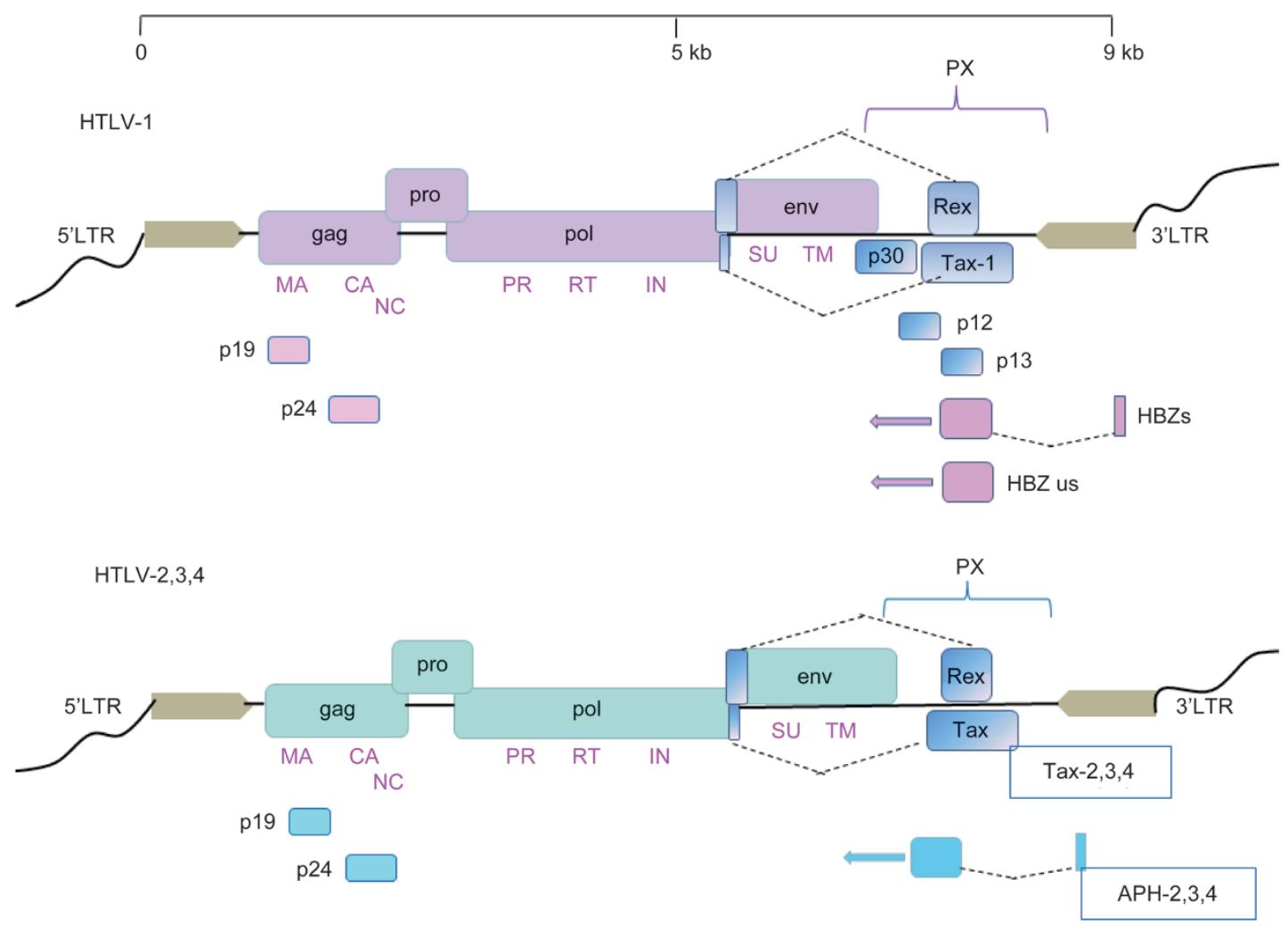

Figure 1. Schematic representation of HTLV-1 and HTLV-2/3/4 genomes. p19 and p24 are the major core proteins of HTLVs. Gray: LTR, Long Terminal Repeat; Coding regions for all major proteins: gag, group-specific antigen; pro, protease; pol, polymerase; env, envelope; rex, regulator of expression; tax, transactivator; HBZ, HTLV basic leucine zipper. The pink letters indicate the positions of the major proteins encoded by the corresponding genes, including the MA (matrix), CA (capsid), NC (nucleocapsid) proteins of gag, the PR (protease), RT (reverse transcriptase), IN (integrase) enzymes of pol and the SU (surface) and TM (transmembrane) proteins of env. 
followed by NRP-1, to form a complex. Then, a fusion process occurs via the combination of GLUT1 with the HSPG/NRP-1 complex ${ }^{[52-54]}$. Afterwards, a viral core containing the viral RNA is delivered into the cytoplasm of the target cells. After reverse transcription, HTLV-1 integrates its genome into the host genome to form a provirus, flanked by two LTRs at the $5^{\prime}$ and $3^{\prime}$ regions. Next, the provirus transcribes and encodes structural regulatory and accessory proteins. Subsequently, the viral genomic RNA and Gag, Env and Gag-Pol proteins are transported to the plasma membrane to assemble into an immature viral particle $e^{[55]}$. The budding particle is then released from the cell surface and undergoes a maturation process to form a mature and infectious viral particle ${ }^{[56]}$. Viral Rex plays an important role in the productive phase of the viral infection and is needed for efficient viral replication. Therefore, Rex is critical for the virus to establish a successful infection $^{[40]}$.

Telomerase activity is required for avoiding replicative senescence and supports immortalization of cells ${ }^{[57]}$. While telomerase activity is absent in normal somatic cells, induction of telomerase expression is a hallmark of cancer cells ${ }^{[58]}$. Telomerase is a holoenzyme consisting of several subunits, such as hTR (human telomerase RNA) and hTERT (human telomerase reverse transcriptase $)^{[59]}$. hTERT acts as a cellular reverse transcriptase and can extend telomeric ends, and therefore, it is the rate-limiting factor for telomerase activity ${ }^{[60]}$. Previous studies have demonstrated that HTLV-1 is able to directly elevate hTERT expression and hTERT activity ${ }^{[61]}$. However, initial findings on the regulation of hTERT by Tax are controversial ${ }^{[61,62]}$. Further experiments have demonstrated that the regulation of the hTERT promoter by Tax is dependent on the cell cycle. Tax positively regulates the hTERT promoter in resting $\mathrm{T}$-cell but negatively regulates it in activated T-cell ${ }^{[63]}$. Tax is able to recruit E2F to a 43-bp region of the hTERT promoter to positively mediate telomerase expression. Tax is also known to stimulate the transcription of two positive telomerase regulators c-myc and Sp1 through the activation of NF- $\mathrm{KB}^{[57,64,65]}$. In addition to Tax, another viral protein, HBZ, has been found to stimulate the transcription of hTERT in a JunD- and SP-1-dependent manner ${ }^{[66]}$. Furthermore, HBZ can degrade the T-cell acute lymphoblastic leukemia 1 protein (Tal1), thus preventing the Tal1-dependent degradation of hTERT mRNA ${ }^{[57]}$. Taken together, these observations indicate that the reactivation of hTERT expression by HTLV-1 contributes to cellular transformation during HTLV-1 infection.

\section{Roles of HTLV-1 genes in tumorigenesis}

HTLV-1 is etiologically linked to adult T-cell leukemia/ lymphoma $(\mathrm{ATL})^{[8]}$. The genetic structure and regulation of HTLV-1 are more complex than those of other leukemia viruses $^{[2]}$. In addition to the structural genes (gag, pro, pol, and env) encoding the characteristic virion proteins, the genome of HTLV-1 also carries genes that encode the nonstructural proteins, Tax and HBZ, which are needed for regulating viral gene expression $^{[38]}$. Although considerable progress has been made in understanding the intricate mechanism of ATL caused by
HTLV-1, further investigation is required to elucidate the function and regulation of the viral gene products and their interactions with one another, as well as with cellular factors.

\section{Tax of HTLV-1 plays an essential role in cellular transformation} and interaction with the host innate immune system

Early studies have shown that Tax-1 is predominantly localized in the nucleus and accumulates specifically in the speckled structures of the nucleus ${ }^{[67,68]}$. Recently, it has been reported that Tax-1 is also localized in the cytoplasm ${ }^{[69-71]}$, although the mechanism regulating the subcellular localization of Tax-1 remains to be elucidated. The $\mathrm{N}$-terminal region of Tax-1 contains a CREB-binding region ${ }^{[72]}$, which is required for its interaction with proteins involved in cell cycle progression, transcription and cell signaling regulation ${ }^{[73,74]}$. Additionally, Tax-1 interacts with cellular factors such as CAMP response element binding protein (CREB) and transcriptional co-activator $\mathrm{CBP} / \mathrm{p} 300^{[75]}$. As a viral oncoprotein, Tax-1 plays a critical role in tumorigenesis and contributes to the pathogenesis of ATL by regulating several intracellular signaling pathways, including IкB kinase (IKK)/NF-кB signaling pathway ${ }^{[76]}$, DNA damage repair pathway, and the innate immune signaling pathways, such as RIG-I/MDA5-dependent and TLR-independent pathways, TRIF-dependent TLR pathways and the recently discovered cGAS-STING pathway ${ }^{[77,78]}$.

Tumor cells are typically characterized by genetic and phenotypic instability, referred to as the mutant phenotype ${ }^{[79]}$. Due to internal (metabolic) and external (genotoxic stress) factors as well as errors in DNA replication, genomic injuries can occur $^{[80]}$. Typically, these errors are corrected immediately by numerous cellular repair mechanisms ${ }^{[79]}$. If these repair pathways cannot be tightly coordinated, the genomic lesions may develop into mutations during cell division and DNA replication, thereby leading to genomic instability. It is thought that a prominent feature of HTLV-1 transformed cells is the genomic instability that is caused by Tax-1-mediated inhibition of the cellular DNA repair pathways ${ }^{[81]}$ and increased mutations in the cellular genome ${ }^{[80]}$. The random nature of these mutations indicates that this viral protein could directly and indirectly interfere with the DNA damage repair pathways, including BER (base excision repair), NER (nucleotide excision repair), MMR (mismatch repair), NHEJ (non-homologues end joining) and HR (homologous recombination). In particular, Tax-1 has been demonstrated to suppress the NER pathway through the transactivation of PCNA (proliferating cell nuclear antigen), a cofactor for DNA polymerase $\delta$ that plays a crucial role in DNA replication and repair ${ }^{[82,83]}$. In addition, by the inactivation of p53, Tax-1 can interfere with the function of the tumor suppressor $^{[84]}$. Tax-1 has a dose-dependent dual effect on NER. High levels of Tax-1 increase the levels of p53 but inactivate p53 function, leading to the inhibition of p53-dependent NER. However, low levels of Tax-1 stimulate NER by increasing the transcriptional activity of $\mathrm{p} 53^{[85]}$.

Additionally, Tax-1 can disturb cell cycle progression, leading to aberrant proliferation of the HTLV-1-infected T-cell ${ }^{[79]}$. Persistent activation of NF-kB is crucial in supporting T-cell 
survival and malignancy. Tax-1 directly targets the beclin1containing autophagy molecular complex to deregulate autophagy by stimulating the IKK complex, resulting in the persistent activation of $\mathrm{NF}-\mathrm{KB}^{[76,86]}$. Autophagy not only has significant roles in preventing inflammation and viral infections but also suppresses tumorigenesis by maintaining chromosomal integrity ${ }^{[87]}$. A previous report has shown that Tax-1 induces the production and secretion of pro-inflammatory cytokines, leading to activation of the IL-6/STAT3 pathway in HTLV-1-transformed T-cell ${ }^{[88]}$. It is thought that when chronic inflammation is present, the risk of tumor occurrence increases.

Viral HBZ promotes cell proliferation and modulates multiple host factors contributing to tumorigenesis

In 2002, a new open reading frame (ORF) was identified in the minus strand of HTLV-1, which encodes a novel basic leucine zipper factor called HBZ ${ }^{[89]}$. HBZ plays indispensable roles in regulating genomic integrity, cell proliferation, apoptosis, autophagy and immune escape ${ }^{[49]}$. HBZ modulates cell growth by forming heterodimers with host factors, such as CCAAT/enhancer binding protein- $\mathrm{a}(\mathrm{C} / \mathrm{EBP} \mathrm{a})$ and activating transcription factor $3(\mathrm{ATF} 3)^{[90]}$. C/EBP a usually acts as a negative regulator of cancer cell proliferation ${ }^{[90]}$. HBZ can overcome the suppressive effect of C/EBP a on cell growth by interacting with $\mathrm{C} / \mathrm{EBP} \mathrm{a}$ and diminishing its DNA binding capacity and by promoting cell proliferation. ATF3, an HBZbinding protein, has bimodal functions in oncogenesis. On one hand, ATF3 activates p53 signaling and acts as a tumor suppressor $^{[91]}$. On the other hand, it promotes cancer cell proliferation $^{[49,91]}$. Interestingly, HBZ inhibits the p53-enhancing function of ATF3, which is deleterious to ATL development, but does not hinder the cell proliferative function of ATF3 in ATL cells ${ }^{[91]}$. Two independent studies have shown that HBZ also employs certain autocrine/paracrine pathways to enhance ATL cell proliferation ${ }^{[92,93]}$. HBZ suppresses the canonical Wnt pathway, but it enhances the proliferation and migration of ATL cells by increasing expression of the noncanonical Wnt5a ${ }^{[92]}$. In addition, HBZ upregulates the expression of brain-derived neurotropic factor (BDNF) and its receptor, tropomyosin receptor kinase B (TrkB), which further promotes ATL cell proliferation ${ }^{[93]}$. HBZ-induced double-strand breaks (DSBs) are dependent on several microRNAs (miRNA) that are HBZ-inducible, such as miR17 and miR21 ${ }^{[94]}$. miR17 and miR21 target and suppress the expression of OBFC2A, a gene that encodes hSSB2, which is a single-stranded DNA-binding protein that prevents genomic instability ${ }^{[94,95]}$. Therefore, HBZ disrupts the genomic integrity of the host through the HBZmicroRNA-OBFC2A cascade ${ }^{[95]}$.

HBZ inhibits apoptosis and autophagy in HTLV-1 infected cells $^{[96-98]}$. A pro-apoptotic gene Bim is greatly suppressed by $\mathrm{HBZ}^{[98]}$. FoxO3a is an important transcription factor that controls the expression of Bim ${ }^{[98]}$. HBZ impairs the DNA-binding ability of FoxO3a and sequesters the inactive, phosphorylated FoxO3a in the nucleus by forming a ternary complex with FoxO3a and 14-3-3, thereby repressing the transactivation of
Bim and leading to the inhibition of apoptosis ${ }^{[98]}$. Autophagy is a natural cellular digestion mechanism that removes unnecessary or damaged cellular components ${ }^{[97,99]}$. Recently, HBZ has been reported to activate the mTOR pathway by inhibiting growth arrest and DNA damage-inducible protein 34 (GADD34), a stress-induced GADD family protein that inhibits the mTOR pathway ${ }^{[100]}$. In addition, autophagy and the mTOR pathway are inversely coupled; thus, mTOR activation caused by HBZ inhibits autophagy ${ }^{[96]}$. Furthermore, it has been shown that among the HTLV-1 viral proteins, HBZ has the lowest immunogenicity because anti-HBZ antibodies can barely be detected in HTLV-1-infected individuals ${ }^{[101]}$. $A$ recent study suggests that the weak binding strength of HBZ epitopes to CTLs and the low expression of HBZ protein might greatly hinder the ability of the host to mount a successful anti-HBZ CTL response ${ }^{[96]}$. It has been suggested that the low immunogenicity of HBZ likely helps the infected cells to escape immunosurveillance and contributes to HTLV-1-mediated oncogenesis.

\section{The clinical diagnoses and therapies of HTLV-1 associated cancer}

ATL is a rare T-cell malignancy caused by HTLV-1 infection. There are four subtypes of ATL (acute, lymphoma, chronic and smoldering) according to clinical manifestations, such as the white blood cell count in blood, serum lactate dehydrogenase (LDH) levels, organ involvement and blood calcium level ${ }^{[102]}$. The clinical diagnosis of ATL is based on the antiHTLV-1 antibody in the serum and the existence of a mature T-cell malignancy. To further confirm ATL, a Southern blot analysis is needed to identify the monoclonal integration of the HTLV-1 provirus into the target tumor cells. Recently, Shigeki Takemoto et al. ${ }^{[103]}$ reported that soluble CD30 (sCD30), a member of the tumor necrosis factor receptor superfamily, is a possible marker for ATL in HTLV-1 carriers. CD30 is expressed in lymphoma and even in normal cells ${ }^{[104]}$, and the soluble form of CD30 is produced by CD30-positive cells ${ }^{[105]}$. Shigeki Takemoto et al found that ATL cells produced SCD30, and elevated levels of serum sCD30 correlated not only with the aggressiveness of the acute type of ATL but also with chemotherapy resistance ${ }^{[106]}$. Furthermore, it has been suggested that the analysis of sCD30 levels may be useful for the prediction of overall survival of ATL patients, especially before allogeneic hematopoietic stem cell transplantation ${ }^{[107]}$. Together, these studies have revealed that the increased circulating sCD30 is associated with the risk of developing ATL in asymptomatic HTLV-1 carriers.

The current treatment strategy for ATL varies from region to region. Additionally, the treatment is based on the clinical subtype ${ }^{[108]}$. Strategies to combat HTLV-1 infections include peptide, recombinant protein, DNA, and viral vectors, and they ultimately lead to the development of various experimental vaccination and therapeutic regimens ${ }^{[109]}$. However, the prognosis continues to be dismal for patients with aggressivetype ATL. To improve treatment outcomes, recent efforts have been focused on the combination of therapeutic modali- 
ties, such as chemotherapy, antiviral therapy, allogeneic hematopoietic stem cell transplantation, and molecular targeted therapy, as well as the development of prognostic stratification and improved dosage and timing ${ }^{[110]}$.

Early chemotherapy is required for patients with aggressive ATL, including the acute and chronic types. To date, several chemotherapy regimens such as CHOP (cyclophosphamide, doxorubicin, vincristine, and prednisolone) and EPOCH (VP16, prednisolone, vincristine, cyclophosphamide, doxorubicin) have been evaluated in patients with aggressive ATL ${ }^{[111]}$. Since 1978, the JCOG-Lymphoma Study Group (JCOG-LSG) has played a particularly central role in the development of chemotherapy regimens for ATL patients. Modified (m)LSG15 (VCAP-AMP-VECP), a new chemotherapy regimen, has replaced one course of VCAP-AMP-VECP from the original LSG15 regimen with intrathecal administration of methotrexate and prednisone as prophylaxis against central nervous system relapse. The original LSG15 regimen consists of sequential administration of vincristine, cyclophosphamide, doxorubicin, and prednisolone (VCAP); doxorubicin, ranimustine, and prednisolone (AMP); and vindesine, etoposide, carboplatin, and prednisolone (VECP). Recently, the mLSG15 as an intensive chemotherapy has been recommended for aggressive ATL ${ }^{[110]}$. It has been suggested that approximately $70 \%$ of ATL cases undergo clonal evolution, and genetic instability may contribute to genomic alterations ${ }^{[112]}$. The existence of multiple clones with genomic instability is a crucial factor that renders ATL cells resistant to conventional chemotherapy. Even if a proportion of cells are killed by chemotherapy, new resistant clones are likely to emerge. For this reason, autologous HSCT (allo-HSCT) might be efficient in curing ATL patients by eliminating the HTLV-1-integrated recipient ATL clones through a strong immune response and subsequent replacement of the hematopoietic system with donor cells ${ }^{[113]}$. Because type I interferons (IFNs) are critical effectors of the innate antiviral response, the combination of alpha interferon and zidovudine, an inhibitor of viral reverse transcriptase, has also been recommended as the standard first-line therapy for ATL.

Moreover, it has been reported that C-C chemokine receptor 4 (CCR4), a seven-transmembrane $\mathrm{G}$ protein-coupled receptor, may be used as a new target for ATL treatment. CCR4 is primarily expressed in regulatory $\mathrm{T}$ (Treg) cells and $\mathrm{T}$ helper type 2 (Th2) cells ${ }^{[14]}$, where it functions in inducing the homing of these leukocytes to sites of inflammation ${ }^{[115,116]}$. Treg impairs the host antitumor immunity and forms a favorable internal environment for tumor growth ${ }^{[12]}$. CCR4 is highly expressed in ATL, and approximately $90 \%$ of HTLV-1-infected individuals are CCR4-positive ${ }^{[117]}$. Remarkably, patients with CCR4positive ATL have worse outcomes than those with CCR4negative ATL. The results suggest that the upregulation of CCR4 plays a key role in the pathogenesis of ATL, likely through Treg functioning. Therefore, therapy with anti-CCR4 antibody may be a good choice. The first-in-class, man-made mogamulizumab, an anti-CCR4 monoclonal antibody, has been approved for the treatment of relapsed/refractory ATL in Japan ${ }^{[118]}$.

Because the viral nonstructural proteins HBZ and Tax are critically involved in HTLV-1-mediated tumorigenesis, they may be used as targets for ATL treatment. Indeed, Sugata et $a l^{[119]}$ generated recombinant vaccine viruses (rVVs) expressing HBZ to determine the immunogenic potential of the viral protein. They found that rVV-expressing HBZ can induce specific CTL responses, and such vaccination partially protects mice from developing T-cell lymphoma. Furthermore, those authors also identified an HBZ peptide (amino acids 157-176) that could be used as a candidate for a peptide-based vaccine $^{[119,120]}$. It has also been found that HTLV-1 infection can trigger chronic activation of the CTL response ${ }^{[121]}$. Additionally, a high titer of anti-HTLV-1 antibodies directed against the Tax protein has been found ${ }^{[122]}$, indicating that Tax is the main immunogen during the viral infection ${ }^{[16]}$. Previous studies have reported that the Tax vaccine protected against HTLV-1 infection in an animal model ${ }^{[123]}$. Recently, a study confirmed that the Tax vaccine is effective in ATL patients ${ }^{[124]}$. The current treatment strategies for ATL are summarized in Table 1.

\section{Future direction}

HTLV-1 is a critical human retrovirus that is linked to human cancer. Recently, viral proteins Tax and Rex of HTLV-1 have attracted a large amount of attention in clinical medicine. Based on their sequence homology, all Tax proteins possess a CBP/p300 binding region. These functional domains show some differences among Tax-1, Tax-2, Tax-3 and Tax- 4 . One of the major differences is related to the lysine residue at position 85 of Tax-1, which is necessary for it to bind CBP/ p300 ${ }^{[51]}$. However, this residue is substituted by an arginine in Tax-2, Tax-3 and Tax $-4^{[125]}$, indicating the functional differences between the Tax proteins. Further investigations of host factors altered in ATL may be helpful in identifying potential targets for effective therapies against this type of leukemia. Moreover, investigations on the subcellular localization of Tax proteins will provide useful information for addressing the mechanisms underlying the interaction of Tax with host factors. It has been shown that Tax oncoproteins perturb IFN production and signaling in HTLV-1-infected cells, and a better understanding of the molecular basis of this process would allow us to develop strategies to counteract Tax and re-establish the innate IFN response against the viral infection. For example, the agonists of both RIG-I and STING were found to have broad-spectrum antiviral effects ${ }^{[126,127]}$. In addition to Tax proteins, Rex plays an important role in HTLV pathogenesis. Further studies are still required to define its precise function.

HTLV-1 infection, a neglected and life-long disease, is currently still incurable and untreatable. The prevention of infection-associated cancers might be able to reduce a substantial proportion of human cancers. To prevent the spread of HTLV-1, a safe and effective vaccine is required. Although HBZ may be considered a useful target for ATL treatment, the efficiency of the recombinant vaccine virus expressing HBZ and HBZ peptide-based vaccine still needs to be further tested in human ATL ${ }^{[119]}$. A previous study indicated that recom- 
Table 1. Treatment strategies for adult T-cell leukemia/lymphoma.

\begin{tabular}{lll}
\hline Smoldering or favorable chronic-type ATL & Unfavorable chronic or acute-type ATL & Relapse or refractory ATL \\
\hline . Asymptomatic patients: Watchful waiting & . Chemotherapy: CHOP, EPOCH, & . Allo-HSCT \\
. Symptomatic patients: Interferon- $\alpha$ (IFN- $\alpha) /$ & LSG15 (VCAP-AMP-VECP) and mLSG15 & . Anti-CCR4 antibody \\
zidovudine (AZT) or watchful waiting & . Allogeneic hematopoetic stem cell & . Vaccine: autologous dendritic \\
. Skin lesions: & transplantation (allo-HSCT) & cells with tax-peptide, HBZ \\
skin-directed therapy: topical steroids, & . Interferon- $\alpha$ (IFN- $\alpha$ )/zidovudine(AZT) & . Promising targeted therapies \\
ultraviolet light and radiation & . VCAP-AMP-VECP+mogamulizumab & and new agents: \\
systemic therapy: steroids, oral retinoids & & arsenic trioxide and IFN $\alpha$, \\
or single agent chemotherapy & & histone deacetylase and \\
& & proteasome inhibitors, \\
& & antiangiogenic therapy, \\
& & bortezomib, survivin, $\beta$-catenin, \\
& syk, and lyn inhibitors, etc. \\
\hline
\end{tabular}

binant proteins derived from the immunogenic sequences of gp46 of the viral envelope had an effect on the induction of efficient immune responses ${ }^{[109]}$. However, one of the limitations of antigenic peptides is their poor immunogenicity ${ }^{[128]}$. To increase immune responses, peptide antigens can be potentiated with polymer-based nanoparticles, such as chitosan (CHT) and trimethylchitosan (TMC), as delivery adjuvants to overcome the limitation. It will be interesting to test whether the recombinant proteins of gp46 might be better candidates for vaccination against HTLV-1 infection ${ }^{[129]}$.

Despite the increase in new therapeutic options, treatment of ATL patients remains challenging. Some promising new agents for ATL are currently in translational research, and several are in clinical trials. Of note, the anti-CCR4 antibody mogamulizumab has been approved for refractory ATL. Moreover, CD30 is an activation marker of lymphocytes, and signaling through CD30 is associated with cell proliferation. A CD30-directed antibody-drug conjugate, brentuximab vedotin, has been assessed in a global phase III trial for ATL chemotherapy. In recent years, cancer immunotherapy has experienced rapid development. The chimeric antigen receptor (CAR)-modified T-cell has shown great promise in cancer treatment ${ }^{[130]}$. The modified $\mathrm{T}$-cell can be redirected to recognize tumor antigens through the expression of CAR. For example, the potency of CD19 CAR-T cells is unprecedented in the field of immunotherapy, particularly in the treatment of acute lymphoblastic leukemia (ALL) and B cell malignancies $^{[131,132]}$. CAR-T might be employed in ATL therapy to recognize specific tumor antigens associated with HTLV-1, but further clinical trials are still needed. Additionally, lenalidomide, an immunomodulatory agent, is being tested in a phase II trial for relapsed aggressive ATL ${ }^{[113]}$. There are additional new agents in ongoing ATL trials, including forodesine (a purine nucleoside phosphorylase inhibitor), pralatrexate (an anti-folate agent), and IL-2 fused with the diphtheria toxin targeting CD25. The application of several inhibitors, such as histone deacetylase inhibitors panobinostat (LBH589), romidepsin, depsipeptide (HFR901228) and the proteasome inhibitor bortezomib, has drawn greater attention in the field of ATL ${ }^{[113,133]}$. However, further studies are required to evaluate the efficacy of these inhibitors and prevent the occurrence of their side effects.

\section{Acknowledgements}

This work was supported by the Natural Science Foundation of China (No U1305212), the National Basic Research Program (973) of China (№ 2015CB910502), and the National Natural Science Foundation of China (№ 31402217 and 81502397).

\section{References}

1 Romanelli MG, Diani E, Bergamo E, Casoli C, Ciminale V, Bex F, et al. Highlights on distinctive structural and functional properties of HTLV Tax proteins. Front Microbiol 2013; 4: 271.

2 Poiesz BJ, Ruscetti FW, Gazdar AF, Bunn PA, Minna JD, Gallo RC. Detection and isolation of type $C$ retrovirus particles from fresh and cultured lymphocytes of a patient with cutaneous T-cell lymphoma. Proc Natl Acad Sci U S A 1980; 77: 7415-9.

3 Van DS, Salemi Mvandamme AM. Dating the origin of the African human T-cell lymphotropic virus type-I(HTLV-I) subtypes. Mol Biol Evol 2001; 18: 661-71.

4 Hajj HE, Nasr R, Kfoury Y, Dassouki Z, Nasser R, Kchour G, et al. Animal models on HTLV-1 and related viruses: what did we learn? Front Microbiol 2012; 3: 333.

5 Hinuma Y, Miyoshi I. Adult T-cell leukemia: antigen in an ATL cell line and detection of antibodies to the antigen in human sera. Proc Natl Acad Sci U S A 1981; 78: 6476-80.

6 Gessain A, Gessain A, Cassar O. Epidemiological aspects and world distribution of HTLV-1 infection. Front Microbiol 2012; 3: 388.

7 Gessain A, Cassar O. Epidemiological aspects and world distribution of HTLV-1 infection. Front Microbiol 2012; 3: 388.

8 Proietti FA, Carneiroproietti ABF, Catalansoares BC, Murphy EL. Global epidemiology of HTLV-I infection and associated diseases. Oncogene 2005; 24: 6058-68.

9 Treviño A, García J, Mendoza CD, Benito R, Aguilera A, Ortíz DL, et al. Prevalence of HTLV-1/2 infections in Spain: a cross-sectional hospital-based survey. AIDS Res Hum Retroviruses 2010; 26: 8614.

10 Gessain A, Mahieux R. Tropical spastic paraparesis and HTLV1 associated myelopathy: clinical, epidemiological, virological and 
therapeutic aspects. Rev Neurol (Paris) 2012; 168: 257-69.

11 Yamagishi M, Watanabe T. Molecular hallmarks of adult T cell leukemia. Front Microbiol 2012; 3: 334.

12 Matsuoka M, Jeang KT. Human T-cell leukaemia virus type 1 (HTLV1) infectivity and cellular transformation. Nat Rev Cancer 2007; 7 : 270-80.

13 Dan LL, Gelmann EP, Cossman J, Young RA, Gallo RC, O'brien SJ, et al. Isolation of HTLV-transformed B-lymphocyte clone from a patient with HTLV-associated adult T-cell leukaemia. Nature 1984; 310: 505-6.

14 Koyanagi Y, Itoyama Y, Nakamura N, Takamatsu K, Kira JI, Iwamasa T, et al. In vivo infection of human T-cell leukemia virus type I in non-T cells. Virology 1993; 196: 25-33.

15 Pique C,Jones KS. Pathways of cell-cell transmission of HTLV-1. Front Microbiol 2012; 3: 378.

16 Carneiroproietti ABF, Amarantodamasio MS, Lealhoriguchi CF, Bastos RHC, Seabrafreitas G, Borowiak DR, et al. Mother-to-child transmission of human T-cell lymphotropic viruses-1/2: what we know, and what are the gaps in understanding and preventing this route of infection. J Pediatric Infect Dis Soc 2014; 3: S24-S29.

17 Percher F, Jeannin P, Martin-Latil S, Gessain A, Afonso PV, Vidy-Roche A, et al. Mother-to-child transmission of HTLV-1 epidemiological aspects, mechanisms and determinants of mother-to-child transmission. Viruses 2016; 8. pii: E40.

18 Kazanji M, Mouingaondémé A, Lekanadoukietenna S, Caron M, Makuwa M, Mahieux R, et al. Origin of HTLV-1 in hunters of nonhuman primates in central Africa. J Infect Dis 2015; 211: 361-5.

19 Tanaka T, Hirata T, Parrott G, Higashiarakawa M, Kinjo T, Kinjo T, et al. Relationship among strongyloides stercoralis infection, human T-cell lymphotropic virus type 1 infection, and cancer: a 24-year Cohort Inpatients Study in Okinawa, Japan. Am J Trop Med Hyg 2016; 94: 365-70.

20 Chen JL, Limnander A,Rothman PB. Pim-1 and Pim-2 kinases are required for efficient pre-B-cell transformation by $\mathrm{v}$-Abl oncogene. Blood 2008; 111: 1677-85.

21 Guo G, Qiu X, Wang S, Chen Y, Rothman PB, Wang Z, et al. Oncogenic E17K mutation in the pleckstrin homology domain of AKT1 promotes v-Abl-mediated pre-B-cell transformation and survival of Pim-deficient cells. Oncogene 2010; 29: 3845-53.

22 Yang J, Wang J, Chen K, Guo G, Xi R, Rothman PB, et al. elF4B phosphorylation by pim kinases plays a critical role in cellular transformation by Abl oncogenes. Cancer Res 2013; 73: 4898-908.

23 Ohshima $\mathrm{H}$, Bartsch $\mathrm{H}$. Chronic infections and inflammatory processes as cancer risk factors: possible role of nitric oxide in carcinogenesis. Mutat Res 1994; 305: 253-64.

24 Huebner RJ, Todaro GJ. Oncogenes of RNA tumor viruses as determinants of cancer. Proc Natl Acad Sci U S A 1969; 64: 1087 94.

25 Vedham V, Verma M, Mahabir S. Early-life exposures to infectious agents and later cancer development. Cancer Med 2015; 4: 190822.

26 Lehky TJ, Flerlage N, Mcfarland HF, Jacobson DS, Katz D, Houff S, et al. Human T-cell lymphotropic virus type II-associated myelopathy: Clinical and immunologic profiles. Ann Neurol 1996; 40: 714-23.

27 Eiraku N, Novoa P, da Costa Ferreira M, Monken C, Ishak R, da Costa Ferreira $\mathrm{O}$, et al. Identification and characterization of a new and distinct molecular subtype of human T-cell lymphotropic virus type 2 . J Virol 1996; 70: 1481-92.

28 Feuer G,Green PL. Comparative biology of human T-cell lymphotropic virus type 1 (HTLV-1) and HTLV-2. Oncogene 2005; 24: 5996-6004.

29 Verdonck K, González E, Van DS, Vandamme AM, Vanham G, Gotuzzo
E. Human T-lymphotropic virus 1: recent knowledge about an ancient infection. Lancet Infect Dis 2007; 7: 266-81.

30 Roucoux DF, Murphy EL. The epidemiology and disease outcomes of human T-lymphotropic virus type II. AIDS Rev 2004; 6: 144-54.

31 Wolfe ND, Heneine W, Carr JK, Garcia AD, Shanmugam V, Tamoufe $\mathrm{U}$, et al. Emergence of unique primate T-lymphotropic viruses among central African bushmeat hunters. Proc Natl Acad Sci U S A 2005; 102: 7994-9.

32 Calattini S, Chevalier SA, Duprez R, Bassot S. Discovery of a new human T-cell lymphotropic virus (HTLV-3) in central Africa. Retrovirology 2005; 2: 1-4.

33 Switzer WM, Salemi M, Qari SH, Jia H, Gray RR, Katzourakis A, et al. Ancient, independent evolution and distinct molecular features of the novel human T-lymphotropic virus type 4. Retrovirology 2009; 6: 253.

34 Gallo RC, Salahuddin SZ, Popovic M, Shearer GM, Kaplan M, Haynes $\mathrm{BF}$, et al. Frequent detection and isolation of cytopathic retroviruses (HTLV-III) from patients with AIDS and at risk for AIDS. Science 1984; 224: 500-3.

35 Gallo RC. Human retroviruses after 20 years: a perspective from the past and prospects for their future control. Immunol Rev 2002; 185 : 236-65.

36 Gallo RC. The early years of HIV/AIDS. Science 2002; 298: 172830.

37 Willems L, Hasegawa H, Accolla R, Bangham C, Bazarbachi A, Bertazzoni $U$, et al. Reducing the global burden of HTLV-1 infection: an agenda for research and action. Antiviral Res 2016; 137: 41-8.

38 Green PL,Chen IS. Regulation of human T cell leukemia virus expression. FASEB J 1990; 4: 169-75.

39 Barbeau B,Mesnard JM. Making sense out of antisense transcription in human T-cell lymphotropic viruses (HTLVs). Viruses 2011; 3: 45668.

40 Younis I, Green PL. The human T-cell leukemia virus Rex protein. Front Biosci 2005; 10: 431-45.

41 Switzer WM, Qari SH, Wolfe ND, Burke DS, Folks TM, Heneine W. Ancient origin and molecular features of the novel human T-lymphotropic virus type 3 revealed by complete genome analysis. J Virol 2006; 80: 7427-38.

42 Calattini S, Chevalier SA, Duprez R, Afonso P, Froment A, Gessain A, et al. Human T-cell lymphotropic virus type 3: complete nucleotide sequence and characterization of the human tax 3 protein. J Virol 2006; 80: 9876-88.

43 Edwards D, Fenizia C, Gold H, De Castro-Amarante MF, Buchmann C, Pise-Masison CA, et al. Orf-I and orf-II-encoded proteins in HTLV-1 infection and persistence. Viruses 2011; 3: 861-85.

44 Nicot C, Dundr M, Johnson JM, Fullen JR, Alonzo N, Fukumoto R, et al. HTLV-1-encoded p30II is a post-transcriptional negative regulator of viral replication. Nat Med 2004; 10: 197-201.

45 Silic BM, Cavallari IN, Vidali S, Chieco BL, Di LF, Saggioro D, et al. Redox regulation of T-cell turnover by the $\mathrm{p} 13$ protein of human T-cell leukemia virus type 1: distinct effects in primary versus transformed cells. Blood 2010; 116: 54-62.

46 Andresen V, Pisemasison CA, Sinhadatta U, Bellon M, Valeri V, Washington PR, et al. Suppression of HTLV-1 replication by Taxmediated rerouting of the $\mathrm{p} 13$ viral protein to nuclear speckles. Blood 2011; 118: 1549-59.

47 Van PN, Gold H, Andresen V, Schwartz O, Jones K, Ruscetti F, et al. Human T-cell leukemia virus type 1 p8 protein increases cellular conduits and virus transmission. Proc Natl Acad Sci U S A 2010; 107: 20738-43.

48 Halin M, Douceron E, Clerc I, Journo C, Ko NL, Landry S, et al. Human 
T-cell leukemia virus type 2 produces a spliced antisense transcript encoding a protein that lacks a classic bZIP domain but still inhibits Tax2-mediated transcription. Blood 2009; 114: 2427-38.

49 Zhao T. The role of HBZ in HTLV-1-induced oncogenesis. Viruses 2016; 8: 130-52.

50 Larocque, Halin M, Landry S, Marriott SJ, Switzer WM, Barbeau B. Human T-cell lymphotropic virus type 3 (HTLV-3)- and HTLV-4-derived antisense transcripts encode proteins with similar Tax-inhibiting functions but distinct subcellular localization. J Virol 2011; 85: 12673-85.

51. Hiramatsu K, Yoshikura H. Frequent partial deletion of human adult T-cell leukemia virus type I proviruses in experimental transmission: pattern and possible implication. J Virol 1986; 58: 508-12.

52 Jones KS, Petrowsadowski C, Bertolette DC, Huang Y, Ruscetti FW. Heparan sulfate proteoglycans mediate attachment and entry of human T-cell leukemia virus type 1 virions into $\mathrm{CD} 4^{+} \mathrm{T}$ cells. J Virol 2005; 79: 12692-702.

53 Manel N, Kim FJ, Kinet S, Taylor N, Sitbon M, Battini JL. The ubiquitous glucose transporter GLUT-1 is a receptor for HTLV. Cell 2003; 115: 449-59.

54 Ghez D, Lepelletier Y, Lambert S, Fourneau JM, Blot V, Janvier S, et al. Neuropilin-1 is involved in human T-cell lymphotropic virus type 1 entry. J Virol 2006; 80: 6844-54.

55 Zhang W, Cao S, Martin JL, Mueller JD, Mansky LM. Morphology and ultrastructure of retrovirus particles. Aims Biophys 2015; 2: 343-69.

56 Konvalinka J, Kräusslich HG, Müller B. Retroviral proteases and their roles in virion maturation. Virology 2015; 479-480C: 403-17.

57 Bellon M, Nicot C. Multiple pathways control the reactivation of telomerase in HTLV-I-associated leukemia. Int J Cancer Oncol 2015; 2.

58 Counter $\mathrm{CM}$. The roles of telomeres and telomerase in cell life span. Mutat Res 1996; 366: 45-63.

59 Cairney CJ, Keith WN. Telomerase redefined: Integrated regulation of hTR and hTERT for telomere maintenance and telomerase activity. Biochimie 2008; 90: 13-23.

60 Hukezalie KR, Wong JMY. Structure-function relationship and biogenesis regulation of the human telomerase holoenzyme. FEBS J 2013; 280: 3194-204.

61 Sinha-Datta U, Horikawa I, Michishita E, Datta A, Sigler-Nicot JC, Brown M, et al. Transcriptional activation of hTERT through the NFkappaB pathway in HTLV-I-transformed cells. Blood 2004; 104: 2523-31.

62 Gabet AS, Mortreux F, Charneau P, Riou P, Ducdodon M, Wu Y, et al. Inactivation of hTERT transcription by Tax. Oncogene 2003; 22 : 3734-41.

63 Hara T, Matsumura-Arioka Y, Ohtani K, Nakamura M. Role of human T-cell leukemia virus type I Tax in expression of the human telomerase reverse transcriptase (hTERT) gene in human T-cells. Cancer Sci 2008; 99: 1155-63.

64 Dittmer J, Pisemasison CA, Clemens KE, Choi KS, Brady JN. Interaction of human T-cell lymphotropic virus type I Tax, Ets1, and Sp1 in transactivation of the PTHrP P2 promoter. J Clin Oncol 1997; 272: 4953-8.

65 Trejo SR, Fahl WE, Ratner L. The Tax protein of human T-cell leukemia virus type 1 mediates the transactivation of the c-sis/platelet-derived growth factor-B promoter through interactions with the zinc finger transcription factors Sp1 and NGFI-A/Egr-1. J Biol Chem 1997; 272: 27411-21.

66 Kuhlmann AS, Villaudy J, Gazzolo L, Castellazzi M, Mesnard JM, Dodon MD. HTLV-1 HBZ cooperates with JunD to enhance transcription of the human telomerase reverse transcriptase gene
(hTERT). Retrovirology 2007; 4: 1-13.

67 Semmes OJ, Jeang KT. Localization of human T-cell leukemia virus type 1 tax to subnuclear compartments that overlap with interchromatin speckles. J Virol 1996; 70: 6347-57.

68 Nicot C, Feng T, Giam CZ. Cytoplasmic forms of human T-cell leukemia virus type 1 Tax induce NF-KB activation. J Virol 1998; 72 : 6777-84.

69 Burton M, Upadhyaya CD, Maier B, Hope TJ,Semmes OJ. Human T-cell leukemia virus type 1 Tax shuttles between functionally discrete subcellular targets. J Virol 2000; 74: 2351-64.

70 Hua C, Cenciarelli C, Shao Z, Vidal M, Parks WP, Pagano M, et al. Human T cell leukemia virus type 1 Tax associates with a molecular chaperone complex containing hTid-1 and Hsp70. Curr Biol 2001; 11: 1771-5.

71 Alefantis T, Barmak K, Harhaj EW, Grant C, WigdahI B. Characterization of a nuclear export signal within the human $T$ cell leukemia virus type I transactivator protein Tax. J Clin Oncol 2003; 278: 21814-22.

72 Yin MJ, Paulssen EJ, Seeler JS, Gaynor RB. Protein domains involved in both in vivo and in vitro interactions between human T-cell leukemia virus type I tax and CREB. J Virol 1995; 69: 3420-32.

73 Suzuki T, Fujisawa JI, Toita M, Yoshida M. The trans-activator tax of human T-cell leukemia virus type 1 (HTLV-1) interacts with cAMPresponsive element (CRE) binding and CRE modulator proteins that bind to the 21-base-pair enhancer of HTLV-1. Proc Natl Acad Sci U S A 1993; 90: 610-4.

74 Goren I, Semmes OJ, Jeang KT, Moelling K. The amino terminus of Tax is required for interaction with the cyclic AMP response element binding protein. J Virol 1995; 69: 5806-11.

75 Kashanchi F, Brady JN. Transcriptional and post-transcriptional gene regulation of HTLV-1. Oncogene 2005; 24: 5938-51.

76 Chen L, Liu D, Zhang Y, Zhang $\mathrm{H}$, Cheng $\mathrm{H}$. The autophagy molecule Beclin 1 maintains persistent activity of NF-KB and Stat3 in HTLV-1transformed T lymphocytes. Biochem Biophys Res Commun 2015; 465: 739-45.

77 Ishikawa H, Barber GN. STING is an endoplasmic reticulum adaptor that facilitates innate immune signalling. Nature 2008; 455: 674-8.

78 Ishikawa H, Ma Z, Barber GN. STING regulates intracellular DNAmediated, type I interferon-dependent innate immunity. Nature 2009; 461: 788-92.

79 Marriott SJ, Semmes OJ. Impact of HTLV-I Tax on cell cycle progression and the cellular DNA damage repair response. Oncogene 2005; 24: 5986-95.

80 Hollingworth R, Grand RJ. Modulation of DNA damage and repair pathways by human tumour viruses. Viruses 2015; 7: 2542-91.

81 Jeang KT, Giam CZ, Majone F, Aboud M. Life, death, and tax: role of HTLV-I oncoprotein in genetic instability and cellular transformation. J Biol Chem 2004; 279: 31991-4.

82 Kao SY, Marriott SJ. Disruption of nucleotide excision repair by the human T-cell leukemia virus type 1 Tax protein. J Virol 1999; 73: 4299-304.

83 Cannon JS, Hamzeh F, Moore S, Nicholas J, Ambinder RF. Human herpesvirus 8-encoded thymidine kinase and phosphotransferase homologues confer sensitivity to ganciclovir. J Virol 1999; 73: 478693.

84 Pisemasison CA, Mahieux R, Jiang H, Ashcroft M, Radonovich M, Duvall $\mathrm{J}$, et al. Inactivation of p53 by human T-cell lymphotropic virus type 1 Tax requires activation of the NF-kappaB pathway and is dependent on p53 phosphorylation. Mol Cell Biol 2000; 20: 337786.

85 Schavinsky-Khrapunsky Y, Priel E, Aboud M. Dose-dependent dual 
effect of HTLV-1 tax oncoprotein on p53-dependent nucleotide excision repair in human T-cells. Int J Cancer 2008; 122: 305-16.

86 Sun SC, Ballard DW. Persistent activation of NF-kappaB by the tax transforming protein of HTLV-1: hijacking cellular IkappaB kinases. Oncogene 1999; 18: 6948-58.

87 Klionsky DJ, Emr SD. Autophagy as a regulated pathway of cellular degradation. Science 2000; 290: 1717-21.

88 Horiuchi S, Yamamoto N, Dewan MZ, Takahashi Y, Yamashita A, Yoshida T, et al. Human T-cell leukemia virus type-I Tax induces expression of interleukin-6 receptor (IL-6R): Shedding of soluble IL-6R and activation of STAT3 signaling. Int J Cancer 2006; 119: 823-30.

89 Gaudray G, Gachon F, Biard-Piechaczyk M, Devaux C, Mesnard JM. The complementary strand of the human T-cell leukemia virus type 1 RNA genome encodes a bZIP transcription factor that down-regulates viral transcription. J Virol 2002; 76: 12813-22.

90 Zhao T, Coutts A, Xu L, Yu J, Ohshima K, Matsuoka M. HTLV-1 bZIP factor supports proliferation of adult $T$ cell leukemia cells through suppression of C/EBP $\alpha$ signaling. Retrovirology 2013; 10: 159.159.13.

91 Hagiya K, Yasunaga JI, Satou Y, Ohshima K, Matsuoka M. ATF3, an HTLV-1 bZip factor binding protein, promotes proliferation of adult T-cell leukemia cells. Retrovirology 2011; 8: 1-12.

92 Ma G, Yasunaga J, Fan J, Yanagawa S, Matsuoka M. HTLV-1 bZIP factor dysregulates the Wnt pathways to support proliferation and migration of adult T-cell leukemia cells. Oncogene 2013; 32: 422230.

93 Polakowski N, Terol M, Hoang K, Nash I, Laverdure S, Gazon H, et al. HBZ stimulates brain-derived neurotrophic factor/TrkB autocrine/ paracrine signaling to promote survival of human T-cell leukemia virus type 1-infected T cells. J Virol 2014; 88: 13482-94.

94 Esquela-Kerscher A, Slack FJ. Oncomirs-microRNAs with a role in cancer. Nat Rev Cancer 2006; 6: 259-69.

95 Vernin C, Thenoz M, Pinatel C, Gessain A, Gout O, Delfaularue MH, et al. HTLV-1 bZIP factor HBZ promotes cell proliferation and genetic instability by activating OncomiRs. Cancer Res 2014; 74: 6082-93.

96 Ma G, Yasunaga Jl, Matsuoka M. Multifaceted functions and roles of HBZ in HTLV-1 pathogenesis. Retrovirology 2016; 13: 1-9.

97 Kim YC,Guan KL. mTOR: a pharmacologic target for autophagy regulation. J Clin Invest 2015; 125: 25-32.

98 Tanaka-Nakanishi A, Yasunaga J, Takai K, Matsuoka M. HTLV-1 bZIP factor suppresses apoptosis by attenuating the function of FoxO3a and altering its localization. Cancer Res 2014; 74: 188-200.

99 Zhou H, Shen T, Shang C, Luo Y, Liu L, Yan J, et al. Ciclopirox induces autophagy through reactive oxygen species-mediated activation of JNK signaling pathway. Oncotarget 2014; 5: 10140-50.

100 Mukai R, Ohshima T. HTLV-1 HBZ positively regulates the mTOR signaling pathway via inhibition of GADD34 activity in the cytoplasm. Oncogene 2014; 33: 2317-28.

101 Enoseakahata Y. Humoral immune response to HTLV-1 basic leucine zipper factor (HBZ) in HTLV-1-infected individuals. Retrovirology 2012; 10: 1-13.

102 Shimoyama M. Diagnostic criteria and classification of clinical subtypes of adult T-cell leukaemia-lymphoma. A report from the Lymphoma Study Group (1984-87). Br J Haematol 1991; 79: 42837.

103 Takemoto S, Iwanaga M, Sagara Y, Watanabe T. Plasma soluble CD30 as a possible marker of adult T-cell leukemia in HTLV-1 carriers: a nested case-control study. Asian Pac J Cancer Prev 2014; 16: 82538.

104 Horie R, Watanabe T. CD30: expression and function in health and disease. Semin Immunol 1999; 10: 457-70.
105 Muta H, Podack ER. CD30: from basic research to cancer therapy. Immunol Res 2013; 57: 151-8.

106 Nishioka C, Takemoto S, Kataoka S, Yamanaka S, Moriki T, Shoda M, et al. Serum level of soluble CD30 correlates with the aggressiveness of adult T-cell leukemia/lymphoma. Cancer Sci 2005; 96: 810-5.

107 Pornkuna R. Clinical value of serum soluble CD30 levels in adult T-cell leukemia/ lymphoma. Am J Hematol 2014; 02.

108 Bazarbachi A, Plumelle Y, Ramos JC, Tortevoye P, Otrock Z, Taylor G, et al. Meta-analysis on the use of zidovudine and interferon-alfa in adult T-cell leukemia/lymphoma showing improved survival in the leukemic subtypes. J Clin Oncol 2010; 28: 4177-83.

109 Lynch MP, Kaumaya PT. Advances in HTLV-1 peptide vaccines and therapeutics. Curr Protein Pept Sci 2006; 7: 137-45.

110 Kato K, Akashi K. Recent advances in therapeutic approaches for adult T-cell leukemia/lymphoma. Viruses 2015; 7: 6604-12.

111 Ratner L, Harrington W, Feng X, Grant C, Jacobson S, Noy A, et al. Human $T$ cell leukemia virus reactivation with progression of adult T-cell leukemia-lymphoma. PLoS One 2009; 4: e4420.

112 Umino A, Nakagawa M, Utsunomiya A, Tsukasaki K, Taira N, Katayama $\mathrm{N}$, et al. Clonal evolution of adult T-cell leukemia/ lymphoma takes place in the lymph nodes. Blood 2011; 117: 54738.

113 Tsukasaki K,Tobinai K. Human T-cell lymphotropic virus type I-associated adult T-cell leukemia-lymphoma: new directions in clinical research. Clin Cancer Res 2014; 20: 5217-25.

114 Tobinai K, Takahashi T, Akinaga S. Targeting chemokine receptor CCR4 in adult T-cell leukemia-lymphoma and other T-cell lymphomas. Curr Hematol Malig Rep 2012; 7: 235-40.

115 Sugata K, Yasunaga J, Miura M, Akari H, Utsunomiya A, Nosaka K, et al. Enhancement of anti-STLV-1/HTLV-1 immune responses through multimodal effects of anti-CCR4 antibody. Sci Rep 2016; 6: 27150.

116 Jean-Michel M, Benoit B, Raymond C, Jean-Marie P. Roles of HTLV-1 basic Zip Factor (HBZ) in viral chronicity and leukemic transformation. potential new therapeutic approaches to prevent and treat HTLV-1related diseases. Viruses 2015; 7: 6490-505.

117 Ishida T, Utsunomiya A, lida S, Inagaki H, Takatsuka Y, Kusumoto $\mathrm{S}$, et al. Clinical significance of CCR4 expression in adult T-cell leukemia/lymphoma: its close association with skin involvement and unfavorable outcome. Clin Cancer Res 2003; 9: 3625-34.

118 Ishida T, Joh T, Uike N, Yamamoto K, Utsunomiya A, Yoshida S, et al. Defucosylated anti-CCR4 monoclonal antibody (KW-0761) for relapsed adult T-cell leukemia-lymphoma: a multicenter phase II study. J Clin Oncol 2012; 30: 837-42.

119 Sugata K, Yasunaga Jl, Mitobe Y, Miura M, Miyazato P, Kohara M, et al. Protective effect of cytotoxic T lymphocytes targeting HTLV-1 bZIP factor. Blood 2015; 126: 1095-105.

120 Mahieux R. A vaccine against HTLV-1 HBZ makes sense. Blood 2015; 126: 1052-3.

121 Goon PK, Biancardi A, Fast N, Igakura T, Hanon E, Mosley AJ, et al. Human T cell lymphotropic virus (HTLV) type-1-specific $C D 8^{+} \mathrm{T}$ cells: frequency and immunodominance hierarchy. J Infect Dis 2004; 189 : 2294-8.

122 Kira J, Nakamura M, Sawada T, Koyanagi Y, Ohori N, Itoyama Y, et al. Antibody titers to HTLV-I-p40tax protein and gag-env hybrid protein in HTLV-I-associated myelopathy/tropical spastic paraparesis: correlation with increased HTLV-I proviral DNA load. J Neurol Sci 1992; 107: 98-104.

123 Ohashi T, Hanabuchi S, Kato H, Tateno H, Takemura F, Tsukahara $\mathrm{T}$, et al. Prevention of adult T-cell leukemia-like lymphoproliferative disease in rats by adoptively transferred T cells from a donor immunized with human T-cell leukemia virus type 1 Tax-coding DNA 
vaccine. J Virol 2000; 74: 9610-6.

124 Suehiro Y, Hasegawa A, lino T, Sasada A, Watanabe N, Matsuoka $\mathrm{M}$, et al. Clinical outcomes of a novel therapeutic vaccine with Tax peptide-pulsed dendritic cells for adult T cell leukaemia/lymphoma in a pilot study. Br J Haematol 2015; 169: 356-67.

125 Chevalier SA, Meertens LMC, Calattini S, Park H, Alhaj AA, Zhou M, et al. The tax protein from the primate T-cell lymphotropic virus type 3 is expressed in vivo and is functionally related to HTLV-1 Tax rather than HTLV-2 Tax. Oncogene 2006; 25: 4470-82.

126 Chiang C, Beljanski V, Yin K, Olagnier D, Ben YF, Steel C, et al. Sequence-specific modifications enhance the broad-spectrum antiviral response activated by RIG-I agonists. J Virol 2015; 89: 8011-25.

127 Sali TM, Pryke KM, Jinu A, Liu A, Iris A, Rebecca B, et al. Characterization of a novel human-specific STING agonist that elicits antiviral activity against emerging alphaviruses. PLoS Pathog 2016; 11: 1-30.

128 Frangione-Beebe M, Albrecht B, Dakappagari N, Rose RT, Brooks $\mathrm{CL}$, Schwendeman SP, et al. Enhanced immunogenicity of a conformational epitope of human T-lymphotropic virus type 1 using a novel chimeric peptide. Vaccine 2001; 19: 1068-81.

129 Amirnasr M, Tafti TF, Sankian M, Rezaei A, Tafaghodi M. Immunization against HTLV-I with chitosan and tri-methylchitosan nanoparticles loaded with recombinant env23 and env13 antigens of envelope protein gp46. Microb Pathog 2016; 97: 38-44.

130 Maus MV, Levine BL. Chimeric antigen receptor T-cell therapy for the community oncologist. Oncologist 2016; 74: 6383-9.

131 Ghorashian S, Pule M, Amrolia P. CD19 chimeric antigen receptor T cell therapy for haematological malignancies. $\mathrm{Br} J$ Haematol 2015; 169: 463-78.

132 Maude SL, Teachey DT, Porter DL, Grupp SA. CD19-targeted chimeric antigen receptor T-cell therapy for acute lymphoblastic leukemia. Blood 2015; 125: 4017-23.

133 Mesnard JM, Barbeau B, Césaire R, Péloponèse JM. Roles of HTLV-1 basic Zip Factor (HBZ) in viral chronicity and leukemic transformation. potential new therapeutic approaches to prevent and treat HTLV-1related diseases. Viruses 2015; 7: 6490-505. 